\title{
EL ANÁLISIS REFLEXIVO Y EL MÉTODO FENOMENOLÓGICO. CONTRIBUCIÓN A LA DETRASCENDENTALIZACIÓN DE LA FENOMENOLOGÍA ${ }^{1}$
}

\author{
The Reflexive ANALYSIS AND the PHENOMENOLOGICAL METHOD. \\ CONTRIBUTION TO THE DETRASCENDENTALIZATION OF \\ PHENOMENOLOGY
}

\author{
Germán Vargas Guillén \\ Universidad Pedagógica Nacional, Bogotá \\ gevargas@pedagogica.edu.co
}

\begin{abstract}
Resumen: Este estudio da cinco pasos. En el primero se caracterizan los orígenes del análisis reflexivo. Bajo el título "origen" se incluyen, principalmente, las motivaciones y el campo de aplicación al cual está referido. En el segundo paso, se presentan las características del análisis reflexivo, sobre todo en términos de supuestos y de la relación del mismo con el canon fenomenológico de estirpe husserliana. En el tercer paso, se valora el alcance del análisis reflexivo como técnica; en cierto modo, se estudia cómo puede operar con y sin la fenomenología como enfoque, teoría o método integral dentro de la investigación. En el cuarto paso, se establece cómo el análisis reflexivo ha encontrado unas alternativas de aplicación, por parte de Lester Embree; sobre todo, se mira el enlace que tiene o puede tener con la individuación al tenor de la lectura de Ideas II de Husserl. Como cierre, en el quinto paso, se establece críticamente el alcance del análisis reflexivo y las posibilidades que ofrece para el desarrollo o continuación de la fenomenología, en el contexto de las vertientes contemporáneas sobre este enfoque.
\end{abstract}

Palabras clave: Técnica fenomenológica. Primera persona. Sentido. Individuación. Mundo de la vida social.

\begin{abstract}
The article is divided into five moments. The first one presents the origins of the reflective analysis; under the title "origin" is included, mainly, the motivations and the scope to which it is referred. The second one refers to the characteristics of the reflective analysis, especially in terms of assumptions and relate to the same lineage husserlian's phenomenological canon. The third one assesses the scope of the reflective analysis as technique; in this way, it explores how can operate with and without the Phenomenology as approach, theory or method integral within the research. The fourth one sets how reflective analysis has found some alternatives of the application by Lester Embree; most of all, the link that has or can have is regarded with to the individuation in way of Ideas II by Husserl. Finally, it sets critically the scope of the reflective analysis and the possibilities for development or continuation of Phenomenology in the context of the contemporary approach in this perspective.
\end{abstract}

Keywords: Phenomenological technique. Firstperson. Meaning. Individuation. Social lifeworld.

\footnotetext{
${ }^{1}$ Este texto ha sido realizado en el marco del Proyecto Ética fenomenológica y formación. Bogotá, CIUP-Universidad Pedagógica Nacional, Código: DED-469-18.
} 


\section{NOTA PREVIA}

El análisis reflexivo es un método. Incluso se puede ver como una técnica. Sea que se lo tome como método o como técnica, su valor radica en que él en sí mismo es una presentación y un ejercicio de fenomenología. La obra de Lester Embree titulada Análisis reflexivo. Una primera introducción a la investigación fenomenológica, traducida en 2003 por Luis Román Rabanaque, es un esfuerzo por mostrar sistemática y progresivamente la práctica fenomenológica. El libro es un manual. Está diseñado para el uso de instructores y estudiantes de fenomenología. La obra está orientada tanto a estudiantes de filosofía como a los estudiantes de otros campos de la investigación en ciencias sociales, en ciencias de la cultura, en ciencias humanas y en ciencias de la salud.

La investigación sobre el análisis reflexivo se enmarca en el campo que se ha denominado fenomenología de la fenomenología. Ésta, en especial, pone su acento en el problema del método. Esta orientación encontró su "fundación" en la VI Meditación Cartesiana (1988) de Eugen Fink. Aunque puede ser un análisis valioso la contrastación dentro de ese contexto, aquí sólo se deja indicada la relación y la temática.

Para los colombianos el libro de Lester Embree y la presencia de él como instructor fue una de las maneras de impulsar el estudio de la fenomenología en el contexto del Doctorado de la Universidad Pedagógica Nacional. Entre los muchos diálogos a los que dio origen se puede destacar el intercambio entre José Francisco Rodríguez Latorre en su artículo "Carta de un positivista a un fenomenólogo" y mi réplica presentada en un artículo titulado "Respuesta a la epístola de un positivista a un fenomenólogo", ambos publicados en 2006 en la revista Cuadernos de Filosofía Latinoamericana. En uno y otro artículo se hace una reseña y una valoración crítica de la obra de Embree.

En lo personal, acudí al análisis reflexivo con miras a la enseñanza del método fenomenológico y valoré, en especial, su contribución en el debate sobre la fenomenología de la fenomenología como lo presento en la Parte I de mi libro Fenomenología, formación y mundo de la vida. Problemas teóricos y metodológicos de la fenomenología publicado en 2012.

Conocí a Lester Embree cuando con Antonio Zirión, Rosemary Rizo-Patrón, Guillermo Hoyos, Roberto Walton, y otros, fundamos el Círculo Latinoamericano de Fenomenología -lunes 16 de agosto de 1999, Puebla, México-; volvimos a 
coincidir en el II OPO-Meeting ${ }^{2}$ - Lima, Universidad Católica del Perú, agosto 16 al 20 de 2005. Luego aceptó gentilmente nuestra invitación como instructor a la Universidad Pedagógica Nacional -Bogotá, 2007. Volvimos a vernos en el Coloquio Latinoamericano de Fenomenología -en Bogotá, Universidad Pedagógica Nacional, de agosto 29 a septiembre 1 de 2007.

La última vez que coincidimos fue en Segovia en la celebración del IV OPOMeeting - septiembre 19 al 23 de 2011. También en esa ocasión se refirió al análisis reflexivo, sólo que en las conexiones entre éste y la fenomenología del aparecer en la obra de Dorion Cairns.

Hablo de Lester como un amigo en la fenomenología y como un incansable investigador y animador de la difusión de la fenomenología a todo lo largo y ancho de este mundo. De hecho, Harry P. Reeder, amigo y émulo de Embree, siempre bromeó diciendo que, si fuera posible, "Lester llevaría la fenomenología a las otras galaxias. $\mathrm{Y}$ a otros mundos posibles". $\mathrm{Y}$ era verdad.

Quiero detenerme en una valoración del análisis reflexivo como vía para la continuación de la fenomenología, como lo hubiera esperado Lester. Y valoro el análisis reflexivo como una de las aristas más visibles de este fenomenólogo audaz, heterodoxo, políglota, amiguero y hombre leal, como si este fuera parte de su más querido legado.

A Lester Embree, paz en su tumba.

\section{LOS ORÍGENES DEL ANÁLISIS REFLEXIVO}

Lester Embree, conspicuo animador de la promoción de la fenomenología, tuvo la fortuna de contar con Dorion Cairns como su maestro - siempre se dirigía a él como su mentor. Entre sus asiduas tareas, llevó a cabo la de ordenar el archivo (Nachlaß) de Alfred Schütz. Este legado reside en la Universidad de Memphis, en el Center for Advanced Research in Phenomenology (CARP) del que Embree también fue un tenaz colaborador. Son muchos los datos relevantes de la vida de Embree. Aquí sólo se indican los que pueden contribuir a un entendimiento del análisis reflexivo.

\footnotetext{
2 O.P.O. es la sigla para The Organization of Phenomenological Organizations, fundada entre otros por Lester Embree. Una de las actividades centrales de esta organización son los encuentros de la referencia. Cf. http://o-p-o-phenomenology.org/
} 
La relación con el legado de Schütz y su posición de metodólogo pusieron a Embree en la vía de una fenomenología orientada hacia el mundo social; y, en general, hacia la comprensión de las experiencias mundano-vitales: la salud, las ciencias del cuidado, la enfermería.

La fenomenología de Schütz enseñó no sólo cómo el vínculo de esta disciplina se entrelaza con la sociología y la psicología, sino que ofreció la claridad de la exposición del ver, de lo dado a la experiencia sin el recurso al ego trascendental.

En cierto modo, el telón de fondo de la conformación del análisis reflexivo es la siguiente pregunta: ¿cómo proceder, paso a paso, para lograr una descripción fenomenológica del orden de la experiencia en el mundo de la vida social? $Y$, complementariamente, ¿cómo contribuir al esclarecimiento de las estructuras del mundo de la vida social? Éstas son preguntas que gravitan sobre la exposición de Embree. El punto central, sin rechazo de la experticia y erudición de los escolares en y de la fenomenología, del análisis reflexivo es: cómo permitir el acceso del neófito a la descripción fenomenológica, a su desarrollo, a su puesta en práctica. En último término, el lema "iA las cosas mismas!" no es para Embree simple tema de expertos; antes bien, es un desafío que se puede tomar sin el paso y sin el peso de todo el recorrido por la escolástica fenomenológica.

Así, en la mira del análisis reflexivo estuvo y está lo que se puede llamar la práctica fenomenológica. El ejecutor del análisis reflexivo, a su vez, tiene que entenderse como un fenomenólogo practicante. Que esto le permita e, incluso, le lleve a este investigador - sea novato, experto o perito - a estar abierto a la confrontación de las descripciones, a su corregibilidad, es parte inherente del método. Que el peso de la erudición puede impedir ese retorno "iA las cosas mismas!" es algo que, en muchos sentidos, deja aludido Embree; y, sin embargo, en el origen de este asunto está la distinción entre el lema fundacional de la fenomenología y el lema "iIr a los textos mismos!" sean de Platón, Aristóteles, Descartes, Kant o Husserl - contra el cual se alzó no sólo el fundador de la fenomenología, sino el Movimiento Fenomenológico, tal como queda descrito por Spiegelberg en su libro The phenomenological Movement: A Historical Introduction publicado en 1982 o como lo cuenta Edith Stein en su libro Estrellas amarillas traducido en 1992.

Si por algo se caracteriza el origen del análisis reflexivo es porque toma el mundo social no sólo como objeto de la mirada sociológica, psicológica y psicosocial -como en parte la lega Schütz-, sino por la clara comprensión de que el 
mundo social es el que vivimos todos y cada uno de los miembros de las comunidades de sentido. Así, por ejemplo, entre los que usamos el transporte público, los funcionarios de éste y los conductores - de buses y de otros vehículos en las vías- se crea una comunidad de sentido como los describe Embree en su artículo "El dónde y el cuándo de las apariciones visuales y auditivas" publicado en el volumen I del Anuario Colombiano de Fenomenología. Y cada uno de los intervinientes en esta escena, a su manera, es un protagonista. Pero, a su vez, ninguna de las perspectivas agota el sentido; antes bien, ofrece una perspectiva, contribuye a comprender el horizonte.

La comunidad de sentido está compuesta por los sujetos que participan en ella. No obstante, cada quién de los intervinientes conserva su carácter de primera persona. Es una posición indelegable. Desde luego, prima la comunicación y se abre la posibilidad del diálogo. Cuanto más implicado el sujeto, tanto más se logra una sintonía y un despliegue de su punto de vista mediante el análisis reflexivo.

El transporte público es un caso relevante. Lo vivimos y llegamos a tener valoraciones de él antes de todo explicitación. El análisis reflexivo entra a jugar un papel significativo cuando no sólo estamos ante la vivencia nuda, sino que damos el paso para establecer no sólo cómo se nos da, sino cuando podemos relativizar nuestra propia perspectiva y verla desde otras posibilidades dentro del mismo horizonte.

De la misma manera, Embree con sus descripciones fenomenológicas muestra nuestra experiencia del cuidado, de nuestro cuerpo, de nuestra salud. La vuelta de Embree a las ciencias del cuidado, al campo de aplicación de la enfermería, muestra la fuerza de este "iVolver a las cosas mismas!". Cierto, el médico y el enfermero tienen mucho que decir sobre nuestro cuidado. Son cultores de unos saberes, de unas ciencias. Al cabo, sin embargo, cada quien, en primera persona, es el agente de la comprensión de sí mismo, de la actuación sobre sí mismo. En un campo de experiencia la variedad de perspectivas de los participantes enriquece el horizonte, lo cual permite también ampliar el sentido sobre nosotros mismos, sobre nuestro cuerpo. Este ensanchamiento del sentido brinda la posibilidad tanto de comprendernos como de actuar sobre nosotros mismos. Aquí tiene su origen el análisis reflexivo: en la íntima comprensión de ser agentes en primera persona, del sentido; de poder entrar en contacto con las visiones de 
los otros; al cabo, de validar el sentido vivido en comunidad, en la generación de una comunidad de sentido.

Un dato, pues, tiene especial relevancia: el análisis reflexivo no comienza por ninguna de las obras de Husserl o de los miembros del Movimiento Fenomenológico. Comienza por cada quien que decide describir su constitución de sentido. $Y$, sobre todo, la fenomenología no es una doctrina, o al menos no lo es en primera instancia. La fenomenología es primordialmente un método. Y, si se quiere, puede ser aprendido como una técnica. Se puede seguir paso a paso. Estos pasos, desde luego, pueden validar el alcance de lo que está dado a cada quien en primera persona.

Sí, puede haber y hay expertos en fenomenología; pero lo que decide la validez del análisis reflexivo no es, bajo ningún criterio, el principio de autoridad. Es en sí la validación intersubjetiva comienzo y fin de la investigación fenomenológica. En su origen, el análisis reflexivo es un campo abierto y quien llegue a él también tiene que abrirse, en muchos sentidos: despojarse de los textos, del principio de autoridad; entender que la suya, propia, es una perspectiva en un horizonte, validable, pero que no es de suyo verdad; disponerse a describir otras perspectivas $y$, en consecuencia, otros sentidos que son diferentes y enriquecen el horizonte.

En síntesis, el origen del análisis reflexivo radica en tomar en serio el lema "iA las cosas mismas!" y en practicar la fenomenología, la descripción, que se basa en el ver -Embree prefiere llamarlo observar. En abandonar la expertocracia. La fenomenología, practicada al amparo del análisis reflexivo, es la que puede ejecutar y ejerce el ciudadano de a pie, común y corriente, el hombre de carne y hueso. En eso radica su accesibilidad; en eso radica su dificultad. Entonces, todo el mundo está en capacidad de practicar la fenomenología. Pero esto no implica que seamos fenomenólogos "espontáneamente" o, en términos del canon fenomenológico, en "actitud natural". Antes bien, es preciso pasar, explícita y conscientemente, de esa actitud a la reflexión, a la "actitud reflexiva". El camino para operar ese tránsito es el análisis reflexivo.

Y para dar inicio a ese trayecto o camino tanto como para llegar a la meta esperada, se puede hacer un plan. La tarea del análisis reflexivo puede ser delineada en cada uno de sus pasos; e, igualmente, puede ser controlada críticamente. De este modo, la investigación fenomenológica no es la de un ego trascendental que, encerrado en el presidio de la conciencia, pueda llegar al eidos, a 
la verdad. Es en la relación con los otros, ante la variedad de perspectivas que configuran el sentido, su validez, las posibilidades de actuar juntos como se valida el conocimiento.

\section{LAS CARACTERÍSTICAS DEL ANÁLISIS REFLEXIVO}

El análisis reflexivo tiene una vocación subjetiva, anclada en la primera persona; no obstante, al mismo tiempo es intersubjetivo, vive de la corregibilidad y de la validación, puesto que no puede darse en ausencia de esos dos polos; la exigencia de claridad está en su base. Todo lo que se expresa a través del análisis reflexivo tiene que remitir a un mundo de la vida vivido, compartido. Su claridad está, en buena cuenta, asociado al referente, a la posibilidad de indicar el campo de remisión. Por supuesto, no es posible avanzar en él sin el lenguaje, sin la lengua, sin las estructuras semánticas y sintácticas; pero no se puede quedar en su esclarecimiento. Como en la Epístola a los Pisones de Horacio, "el lenguaje es el uso" y, en consecuencia, las dificultades de o en la comunicación se resuelven pragmáticamente, esto es, por referencia al correlato, a la experiencia, al darse del mundo.

Una palabra debe tomarse con especial atención: análisis. Desde luego, remite a la larga tradición filosófica, también matemática, en la que un todo se descompone en sus partes. El todo es la vivencia, pero ésta sólo se nos da en estructuras de sentido. De lo que trata el análisis es de vivencias de sentido que tienen que ser descompuestas en sus elementos constitutivos; pero un "ingrediente" de cada vivencia es su génesis, su devenir. Así, analizar no es sólo un asunto de descripción de un haz de elementos que convergen en una vivencia hasta que se torna en unidad de sentido; es, igualmente, dar cuenta del devenir, de cómo se llega a dar sentido, de cómo se inserta éste en tradiciones y contextos culturales con su haber sido, su ser y su poder llegar a ser. En términos canónicos, la descripción acontece en y desde la temporalidad, con su haz de retenciones y protenciones, siempre en y desde un presente viviente - hic et nunc.

De manera paradojal, el análisis es posible porque, originariamente, la experiencia se nos da como un todo unido, enlazado. En fin, la experiencia, y más exactamente, el sentido que se nos ofrece en ella aparece en el modo de una síntesis. A ésta hemos llegado activamente, por experiencias o vivencias concretas, por enunciados que nos permiten darles sentido; en fin, activamente se ha 
constituido una síntesis - síntesis activa- que se convierte para todos y cada uno de nosotros en un trasfondo - background-, una suerte de sobreentendido. Al punto incluso que ese trasfondo opera sin que nos demos cuenta, en diversas formas: prejuicios, juicios; nociones, categorías, conceptos; valores, normas, hábitos. En fin, ese trasfondo es efecto, se ofrece también como una síntesis. Sólo que ésta opera pasivamente. El trasfondo es pasividad, síntesis pasiva.

La función del análisis es poner al descubierto esta doble síntesis. De preferencia, pasar de la síntesis pasiva en que se está precomprendido el mundo al haz de síntesis activa que vivencial o experiencialmente ha ofrecido la base para la configuración del trasfondo, en fin, de lo que comporta nuestra visión de mundo. El punto radica en que el análisis opere sobre lo enlazado y dé cuente de su constitución. Ésta sólo puede entenderse en el dinamismo del devenir, de lo que se ha vivido o experienciado, en primera persona, por cada quien; y sólo puede ser entendida en esta dimensión. Sólo así se abre el campo de la validez intersubjetiva.

El análisis implica una vuelta a sí mismo, en su posición actual, en su presente viviente, sobre lo vivido. Así, entonces, es el yo en su presente viviente quien opera - reflexivamente- sobre su pasado yo, sobre su devenir. $Y$ al tratar de comprender su ser, su haber sido, su poder llegar a ser: algo se le escapa y algo le agrega. Por lo tanto, la tarea del análisis radica en una suerte de profilaxis que limpia o despeja el campo de la experiencia vivida. De ahí que su recurso para poder acceder a ese campo sea, estructuralmente, la descripción. Para Embree no se trata de narrar, mucho menos de interpretar, puesto que el sujeto se lanzaría a la ficción; tampoco de argumentar, puesto que el sujeto se proyectaría, básicamente, a un proceso de justificación de sus acciones, de sus comprensiones, de sus maneras de asumir el mundo y de entenderse dentro de éste. La descripción, en cambio, puede ser "plana" si se quiere; pero tiene la posibilidad de ser controlada como un despliegue de la comprensión del que es posible dar cuenta, explicitar referencias - de nuevo, se acude al "referente"-, validar mediante dispositivos - la cronología, la historia; el hecho; el dato; etc.

Al cabo, si por algo se caracteriza el análisis en el análisis reflexivo es porque vincula y refiere permanentemente la cosa misma: una vivencia, una experiencia, una materia de hechos, etc. Es, dicho con expresiones del canon fenomenológico, el polo noemático. 
Ahora bien, el análisis no puede ser desconectado de la reflexión. Así, pues, tanto valor tiene el uno como la otra. Ahora es posible detener la atención en esta última. La reflexión pone en juego el campo de las variaciones. Es, en sí, apertura y despliegue del polo noético. No se trata sólo de lo que se da, sino de la manera como se nos da. No sólo es el sentido, sino la diáspora de posibilidades de sentido que se abren cuando vivimos, cuando tenemos una experiencia. No es sólo la manera en que lo entendemos, sino los múltiples horizontes en los cuales puede ser entendido y realizado el sentido.

Sólo en la reflexión, sólo reflexivamente, lo que es así, según una manera de entender una vivencia, una experiencia o un sentido: pudiera ser de otra manera. Es la apertura a mundos posibles. Entonces, los otros sentidos no sólo son posibles, sino que dan apertura a la comprensión de otros, que entienden el mundo de maneras diferentes a nosotros. Es, en nosotros, en primera persona para cada uno de nosotros, la experiencia de la condición de intersubjetividad del sentido: "así lo vivo yo con respecto a esta experiencia, pero se podría vivir asá... y de otras maneras". No es un campo ficcional: no es como si lo estuviera viviendo, sino como si lo pudiera vivir. En una expresión de Husserl: "Llevo a los otros en mí"3, los porto reflexivamente, se me dan las posibilidades de su experiencia.

La reflexividad es la condición de intersubjetividad que me abre a las experiencias que no tengo hic et nunc, pero que pudiera vivir. Entonces, por su naturaleza, la reflexividad desdogmatiza, rompe el curso de la actitud natural, relativiza el punto de vista propio; y, sin embargo, no implica el abandono de lo que efectivamente se me da. Por la reflexividad hay visiones alternativas de mundo. Sobre éstas son posibles tanto disensos como consensos. Por la vía de la reflexividad se abre el campo de las variaciones imaginativas.

El análisis reflexivo es la conjunción estructural de polo noemático y polo noético, tanto de la experiencia de la primera como de la segunda persona. Así, pues, en él sólo cabe la posibilidad de un sentido detrascendentalizado: no hay lugar para el ego solitario. Si por algo se caracteriza el análisis reflexivo es porque no es trascendental. Esta característica lo acerca cada vez más a la idea de ciencia rigurosa y es un "tipo" de método de investigación que puede vérselas con cualquier demanda tanto de rigor como de validez, de contrastación y de recurso p. 139

3 Julia V. Iribarne, La intersubjetividad en Husserl. Vol I, Buenos Aires: Editorial Carlos Lohlé, 1987, 
al referente. Sólo que es un método natal de la fenomenología entendida como ciencia y de entraña personal, social y cultural. De esta manera, característico del análisis reflexivo es que se define por su estructura metodológica y deja de lado cualquier pretensión de doctrina.

Si bien es cierto que Embree mantiene toda esta fundación o fundamentación del análisis reflexivo, podemos decir, en lo más entrañable del canon fenomenológico, su manera de presentarlo se atiene a un lenguaje llano, directo. Por lo tanto, él Ilama observar - como ya se dijo- al "ver" que dejó establecido Husserl desde las Lecciones de 1907 compiladas en la Hua II, mantiene la estructura de ésta como fundante de la práctica fenomenológica. Este observar da lugar al informar, en el cual retoma la sentencia canónica de Husserl según la cual "el mundo de la vida se nos da lingüísticamente sedimentado". Sólo tras estos dos pasos pone el énfasis en el reflexionar que, como se ha mostrado, abre el campo de las variaciones imaginativas. Pero, ¿sobre qué recae todo este proceder? Ahí es cuando Embree da con el querer, el valorar y el creer, en fin, todo lo que en pasividad ha llegado a ser efecto de la actividad. Así, sienta las bases para que metódicamente se tome la dirección del analizar, esto es, el poder dar cuenta de la experiencia producida por sus procesos constitucionales. Este recorrido concluye, como lo muestra Embree, con el problema de la validez, al amparo de lo que llamó el examinar.

\section{EL ALCANCE DEL ANÁLISIS REFLEXIVO COMO TÉCNICA}

A lo largo de su exposición, en su libro Análisis reflexivo, Embree muestra que la fenomenología no es cosa de erudición. De hecho, la llama el "primer vicio". Para él, la erudición incluye "la edición, interpretación, reseña y traducción"4. Tampoco acepta que la fenomenología sea cosa de "argumentación", puesto que los "fenomenólogos genuinos [...] no presentan por regla general argumentos. Presentan más bien «análisis»"5.

Para Embree uno de los hechos más significativos que se debe registrar es que "la fenomenología" no "es exclusivamente una tradición dentro de la filosofía,

${ }^{4}$ Lester Embree, Análisis reflexivo. Una primera introducción a la investigación fenomenológica. A First Introduction into Phenomenological Investigation, trad. Luis Rabanaque, Morelia: Jitanjáfora, 2003, pp. 10 s.

${ }^{5}$ Ibid., pp. 12s. 
es decir, de la filosofía concebida como una especialidad a la par de otras" ${ }^{16}$. En cambio, hay "una corriente fenomenológica en la psiquiatría desde antes de la Primera Guerra Mundial, y recientemente ha resultado fácil identificar tendencias fenomenológicas en una veintena de otras disciplinas no filosóficas durante el primer siglo de la fenomenología"7.

Embree escribió "Prefacio para los instructores"8 a su obra. Este dato es especialmente significativo a la luz de la tesis aristotélica según la cual propio de la ciencia es que puede ser enseñada. Ése es el motivo central de la obra. Por eso, según sus propias palabras: "Los colegas pueden apreciar mi placer por emplear la forma de manual"9. En resumidas cuentas, Embree procede con la fenomenología como si fuera una técnica. Así había sido llamada por Levinas en los Cuadernos Royaumont de 1959 (traducidos por Podetti en 1968).

A diferencia de Levinas, el logro de Embree consiste en que no sólo nombra la fenomenología como técnica, sino que hace un manual para que esta técnica pueda ser estudiada, apropiada, sometida a usos en diversos campos del saber. Por eso el libro no sólo consta de gráficos que sintetizan lo que expone tanto conceptual como operativamente, sino que cuenta con recuadros que podrían o deberían ser resueltos por los aprendices. Igualmente, el libro ofrece recuadros con preguntas que pueden hacer tanto las veces de control de lectura como de ejercicios de aprendizaje. Un caso es el que ofrece la figura 7.1.10, en la sección sobre el examinar; el aprendiz debe responder:

1. ¿Cómo está dispuesto a querer u obrar en esta actitud?

2. ¿Qué es valorado en forma positiva o negativa, absoluta o comparativamente, etc.?

3. En dicha actitud, ¿en qué cosas se cree y de qué modo?

4. ¿Qué tipo de experiencias y de objetos en tanto que experiencias son inherentes a esta actitud?

Didácticamente llama la atención cómo el cuadro comienza con la pregunta 4 que sólo puede ser respondida tras la elaboración de las tres preguntas precedentes. Se trata de aprender a examinar actitudes. Están implicadas la "natural"

\footnotetext{
${ }^{6}$ Ibid.

${ }^{7}$ Ibid.

${ }^{8}$ Ibid., pp. 10-29 y pp. 11-30.

${ }^{9}$ Ibid., pp. 28s.

${ }^{10}$ Ibid., pp. 500 s.
} 
y la "reflexiva", pero ante todo lo que se quiere es que el aprendiz identifique cómo operan, si se quiere, pasivamente, las actitudes como un flujo o una corriente y cómo éstas han llegado a ser constituidas.

Embree expresa confianza en que: llegar a ser un fenomenólogo competente es asunto de práctica, más que de cualquier otra cosa; en que la fenomenología puede ser, como análisis reflexivo, practicada en cualquier campo del saber, siempre que esté implicado el sujeto, en primera persona, en su relación con los otros. De esta manera, el alcance de la técnica está en que pueda ser puesta y se ponga en juego. Incluso, como si se tratara de cualquier otro dispositivo, digamos de un artefacto, la fenomenología puede ser puesta en funcionamiento. Si se siguen las instrucciones, se puede operar con ella. No se exige hacer un voto de confianza en la fenomenología como doctrina; aun si se toma como técnica, quien la practica no tiene que empezar y terminar sus estudios en y por la fenomenología. Antes bien, puede acudir a ella como si fuera un módulo, una fase, un momento o un procedimiento dentro de un diseño que, en últimas, suscribe otro enfoque, otra teoría u otro método.

Si se quiere, es la detrascendentalización de la fenomenología en el modo de una heterodoxia nacida desde el seno mismo del canon. Embree no asume que el análisis reflexivo sea única y exclusivamente proveniente de Husserl, con todo y el santo y seña del canon husserliano. En cambio, asume que en éste conjuga elementos que tributan al pensamiento y la obra de Henri Bergson, William James, Edmund Husserl, Max Scheler, Martin Heidegger, Alfred Schütz, Maurice Merleau-Ponty, Jean-Paul Sartre y de sus maestros Edward G. Ballard, Dorion Cairns y Aron Gurtwitsch ${ }^{11}$.

Por esto, el libro Análisis reflexivo comporta una dosis, sí, muy significativa de "las fenomenologías", pero igualmente tiene acentos y énfasis que provienen del pragmatismo. Si el diálogo entre la fenomenología y el pragmatismo avanza en un punto -más por las cosas mismas que por la erudición - es a raíz de esta obra y, en especial, por el valor que tiene el referente, sea que se lo llame cosa misma, materia de hechos, vivencia, experiencia, sentido.

Tal vez el fenomenólogo canónico, ortodoxo, se pueda prevenir ante el uso de la expresión "técnica" y "técnica fenomenológica". Lo que logra el manual de

\footnotetext{
${ }^{11}$ Ibid., pp. 26s.
} 
Lester Embree es resignificar el valor de estas expresiones. Con ello, una apertura al campo de la investigación en las ciencias sociales, en las ciencias humanas, en las ciencias de la cultura, en las ciencias del cuidado. Este camino es el de una desacralización de la fenomenología, un intento de superar en ella la "expertocracia". La vuelta "ia las cosas mismas!" es la condición de posibilidad de un diálogo interdisciplinario que se nutre de una suerte de llave maestra, a saber, la descripción. Ésta puede ser llevada a cabo "técnicamente". También puede ser corregida "técnicamente". Por lo tanto, se puede tener un diálogo entre diversos sujetos y disciplinas a raíz de las cosas mismas.

Embree, con su libro - con la exposición y la aplicación del análisis reflexivo como "técnica" - cambia el sentido de oposición entre la fenomenología y las diversas expresiones teóricas y metodológicas de las ciencias sociales, de las ciencias de la cultura, de las ciencias humanas, de las ciencias del cuidado - que se engloban, a veces sin los matices necesarios, como positivistas - y abre un plano de cooperación y de diálogo que puede llevar a que éstas se enriquezcan mutuamente. De la misma manera, esta cooperación propicia que la fenomenología se convierta en un proyecto de ampliación de la racionalidad científica al amparo de la perspectiva de los sujetos y de su experiencia en el mundo de la vida.

Sea o no un propósito deliberado de Embree, el análisis reflexivo se abre como un campo de intersección entre la fenomenología y las otras prácticas de investigación que tienen por centro la experiencia humana de mundo, su racionalización, la ampliación de sus sentidos.

\section{LOS CAMPOS DE APLICACIÓN}

Fenomenología continuada. Contribuciones al análisis reflexivo de la cultura, publicada en 2007, es una obra que compila ensayos de Embree de distintos períodos de su trayectoria investigativa. En efecto, lo que ofrece son, puede decirse, aplicaciones: en su primera parte, a las diferencias generacionales, a la etnofobia norteamericana, a las actitudes de clase, a la dialectología de género, a las ficciones de cortesía, a la buena salud de los ecosistemas, bajo el supuesto de que se trata de dimensiones poco exploradas en el "mundo de la vida"; en la segunda parte, en cambio, con una orientación hacia las reflexiones filosóficas, 
atiende: la constitución de la cultura básica, la reflexión sobre las disciplinas culturales, el modelo gurwitscheano para explicar la cultura y los avances concernientes a la valoración y a la acción -según Ideas II de Husserl.

¿Qué es lo que le interesa a Embree, en términos de la aplicación del análisis reflexivo? Se puede responder, sumariamente: 1) "[...] mostrar que el famoso mundo de la vida de la fenomenología es completamente sociocultural"; y, 2) "[...] ver cómo la fenomenología puede ser continuada"12. Sin embargo, desde el punto de vista teórico, tal vez la mayor contribución se encuentra cuando Embree observa que en todo útil -trae a colación el ejemplo de un martillo para abrir nueces - puede ser "analizado en términos de hábitos individuales, existen modelos culturales de acción en lo que participa una multitud de personas"13; en fin, el punto que enfatiza es que "la vida cultural es más práctica que valorativa o cognoscitiva"14. Para Embree, lo que implica la constitución de una "cultura básica" es reconocer que "hay un estrato en la vida consciente y también en el mundo que está por debajo del de las formas categoriales, de los constructos del sentido común, del pensamiento o la interpretación, y que, a la vez, está por encima de la percepción sensible de las cosas naturales"15. Aquí entra en juego la visión de la individuación, del fenómeno y de la fenomenología de la individuación, pues "este estrato puede llamarse "cultura básica", y puede ser abstraído junto con el estrato categorial para alcanzar el nivel de las cosas naturales, las cuales, a propósito, son físicas, vitales o biológicas, o somatopsíquicas"16.

La tesis fuerte del análisis reflexivo es que la cultura -en particular, lo que, según este enfoque, se tiene que entender como "cultura básica"- es el entorno de individuación. Que ésta ocurra en el orden essendi tanto como en el cognoscendi es cosa que se evidencia a cada paso. Sólo que su múltiple darse, en uno y otros ámbitos, puede ser simultáneo o sucesivo; puede darse el primero sin que llegue a conocerse; puede darse en el orden cognoscendi $y$, por éste, impactar o implicar el orden essendi.

La pura fenomenidad es física. Si ésta llega a desplegar una fenomenalización es por la vida, por el despliegue de los procesos biológicos. No obstante, el campo

\footnotetext{
${ }^{12}$ Lester Embree, Fenomenología continuada. Contribuciones al análisis reflexivo de la cultura, Morelia: Jitanjáfora, 2007, p. 9.

${ }^{13}$ Ibid., p. 173.

${ }^{14}$ Ibid., p. 174.

${ }^{15}$ Ibid., p. 176.

${ }^{16}$ Idem.
} 
fenomenológico se abre cuando entra en juego la individuación psíquica, que jamás deja de ser somática. Por eso, puede decirse, el campo fenomenológico es el de la individuación somatopsíquica.

¿Cómo es el orden de esta individuación? En el orden somatopsíquico se da tanto la dimensión pática como la práxica. Es en éstas donde se constituye el valor. "Aquello que tiene un valor positivo puede fácilmente decirse no sólo que es bueno, sino también, como un positum, que es un bien"17. Este bien es lo propio, lo que se da en propiedad. No es una "hacienda" o una "extensión". El bien como positum es lo que aparece o se da; y aparece o se da en la forma de un valor. Lo vivido es tal en la medida en que es valorado, en que es un bien, en que se da en propiedad. Aquí opera la individuación como estructura fundante del orden somatopsíquico.

Embree lee Ideas II de Husserl para ver cómo se integran esos niveles de individuación. En fin, su aproximación muestra cómo el mundo de la cultura, que es entorno de individuación, se erige sobre los estratos biológicos y físicos como su condición de posibilidad. Si el mundo de la vida se da lingüísticamente sedimentado es en razón de su soporte en el estrato biológico y en el físico, que se individúan en la pasividad del modus essendi.

En resumidas cuentas, el campo de aplicación del análisis reflexivo está, al amparo de la lectura de Ideas II, en la dación física y biológica de la hylé. Estas daciones son la condición de posibilidad de la dación de la cultura; y de la operación de la cultura como entorno de individuación.

Para Embree uno de los lugares en que se ve este operar es en las valoraciones relativas a los ecosistemas y la salud de los mismos:

La complejidad de la materia puede enfocarse al nivel de los fines y de los medios que han de ser queridos hacia el bien de la salud general y a través de la consideración de los medios - por ejemplo el tiempo y el esfuerzo - y fines que están más allá de él -como por ejemplo ser medioambientalmente ejemplar en la sociedad. ${ }^{18}$

En estas valoraciones no sólo está en juego la hylé, sino el sentido constituido sobre la misma -las habitualidades, las creencias, los valores, etc.

17 Ibid., p. 236.

18 Ibid., p. 153. 


\section{LIMITACIONES DEL ANÁLISIS REFLEXIVO}

Embree es plenamente consciente de que: "La fenomenología es un enfoque que puede describirse muy concisamente como análisis reflexivo"19. Más aún, que "este enfoque puede ser emprendido en muchas disciplinas"20. Empero, según su punto de vista, "no puede adoptarse en las disciplinas formales tales como en las matemáticas o en las ciencias naturalistas, como la química, aunque puede adoptarse en los esfuerzos posteriores para entender lo que se hace en dichas disciplinas"21.

En todo caso, para Embree, el análisis reflexivo "efectivamente puede ser llevado a cabo, sin embargo, es en las disciplinas que tematizan aspectos del mundo sociocultural"22, a fin de responder a las cuestiones: "Cómo un mundo es cultural en virtud de la valoración y la volición y cómo puede ser social al tener en él a otros, no humanos y humanos"23.

Al menos dos indicaciones deben ser hechas: 1) El análisis reflexivo no da cuenta de cómo es posible la fenomenología en campos de las ciencias que no tematicen manifiesta y directamente la cultura, el mundo social. 2) Al cabo, el análisis reflexivo se las ve con los valores, la volición, las relaciones entre los humanos y de los humanos con su entorno. Su alcance, como queda visto, es al mismo tiempo su limitación. Es canónico en la obra de Husserl y en la tradición fenomenológica el volver la atención a la geometría, la lógica, a las matemáticas. En principio, éstos no son campos del análisis reflexivo; o, al menos, no lo es porque en este reino formal no entra en juego la experiencia relativa al mundo social, al mundo de la cultura; a los valores, a la voluntad. En cierto modo, el análisis reflexivo es una fenomenología única y exclusivamente del mundo social y cultural.

Como queda visto, tampoco está en discusión el espacio. Estudios abiertos por obras como Cosa y espacio - editados en la Hua XVI- quedan, igualmente, al margen de las consideraciones explícitas del análisis reflexivo. Con esto, es una fenomenología que prioriza el tiempo, la vivencia del tiempo, el mundo social y cultural de la temporalidad. Pero, ¿qué pasa con el sistema de los lugares?

\footnotetext{
${ }^{19}$ Lester Embree, Análisis reflexivo. Una primera introducción a la investigación, pp. 194s.

${ }^{20}$ Idem.

${ }^{21}$ Idem.

${ }^{22}$ Idem

${ }^{23}$ Ibid., pp. 94s.
} 
¿Cómo se da la constitución de éste a partir del cuerpo? Y, ¿cómo una fenomenología del cuerpo, una fenomenología del lugar, una fenomenología del espacio - por separado o todas ellas en conjunto - permiten fundar la experiencia intersubjetiva y, en último término, la comprensión del sentido? Todo esto, al parecer, escapa al análisis reflexivo.

Queda todavía un asunto más que no es del todo claro, a saber, si el análisis reflexivo puede estar al margen, es decir, por fuera de toda consideración; o antes bien, en las márgenes de lo psicológico, del estudio de las estructuras de la mente. Tal vez, todavía, el campo de la fenomenología de la mente y la fenomenología de la cognición no han dado cuenta de los procesos idiosincrásicos y de la relación entre estas estructuras y las estructuras del mundo de la vida social y cultural; de la mutua interrelación entre fenomenología cognitiva y/o fenomenología del mundo de la vida social y cultural. El análisis reflexivo puede estar o lo está, en la versión de Embree, al margen. Nuestra hipótesis es que tanto el análisis reflexivo como la fenomenología de las estructuras del mundo de la vida social y cultural, à la Schütz o à la Embree, respectivamente, contribuyen a un atemperamiento de la naturalización de la fenomenología que puede dar con una visión rica de la cognición, de la psicología fenomenológica ${ }^{24}$, de la comprensión no sólo encarnada (embodiment), sino que se valga de la distinción entre cuerpo y carne, que profundice la diferencia entre Körper y Leib, que se comprometa tanto con la fenomenología material como con la fenomenología de la individuación.

La riqueza del análisis reflexivo, tal como lo desarrolla Embree y en lo que es continuador, en buena cuenta, de la tradición abierta por Cairns-Schütz se halla ante una autolimitación que confina la fenomenología a un enfoque sociologista y culturalista. No obstante, queda abierto, por vía de hipótesis, una relectura de la fenomenología como análisis reflexivo en dirección de, al menos, cuatro diálogos: con las ciencias formales y el reino de las estructuras de pensamiento; con las ciencias naturales, y el reino de las dimensiones fisiopsicológicas de los procesos de pensamiento; con las estructurales materiales -la hylé, el espacio, el

24 Cf. Stee Hernández, ¿Es posible naturalizar la fenomenología? La naturalización de la psicología fenomenológica en sentido débil y la imposibilidad de la naturalización en sentido fuerte [Tesis de grado, presentada en la Universidad Pedagógica Nacional, Bogotá, en 2017]. 
sistema de los lugares - que subyacen fenoménicamente a toda posibilidad de la fenomenología; con la individuación física, biológica y psíquica.

El análisis reflexivo está ante la posibilidad de reconocer un reduccionismo metodológico para el inicio de su sistematización y formalización; pero, igualmente, está ante la posibilidad, potente, de generar su resignificación en diálogo con "las fenomenologías" que forman parte de la contemporaneidad y que enriquecen el Movimiento Fenomenológico.

\section{REFERENCIAS}

EMBREE, Lester, Análisis reflexivo. Una primera introducción a la investigación fenomenológica, trad. Luis Rabanaque, Morelia: Jitanjáfora, 2003.

-, Reflective Analysis. A First Introduction into Phenomenological Investigation, Bucarest: Zeta Books, $2011^{2}$.

-, Fenomenología continuada. Contribuciones al análisis reflexivo de la cultura, Morelia: Jitanjáfora, 2007.

- $\quad$ "El dónde y el cuándo de las apariciones visuales y auditivas", Anuario Colombiano de Fenomenología, vol. I (2007) 171-178.

FINK, Eugen, VI Cartesianische Meditation. Teil I: Die Idee einer transzendentalen Methodelehre, Ed. G. van Kerckhoven / H. Ebeling / J. Holl, The Hague: Kuwer Academic Publishers, 1988.

HoRAcio, Epístola a los Pisones (trad. T. Iriarte), en http://www.cervantesvirtual.com

HUSSERL, Edmund, Die Idee der Phänomenologie. Fünf Vorlesungen, ed. Walter Biemel, The Hague: Martinus Nijhoff, 1973. (Hua II).

-, Ideen zur einer reinen Phänomenologie und phänomenologischen Philosophie. Zweites Buch: Phänomenologische Untersuchungen zur Konstitution, ed. Marly Biemel, The Hague: Martinus Nijhoff, 1952. (Hua IV).

—, Ding und Raum. Vorlesungen 1907, ed. Ulrich Claesges, The Hague: Martinus Nijhoff, 1973. (Hua XVI). 
HERNÁNDEZ, Stee, "¿Es posible naturalizar la fenomenología? La naturalización de la psicología fenomenológica en sentido débil y la imposibilidad de la naturalización en sentido fuerte" [Tesis de grado, presentada en la Universidad Pedagógica Nacional, Bogotá, en 2017].

IRIBARNE, Julia V., La intersubjetividad en Husserl: Vol I, Buenos Aires: Editorial Carlos Lohlé, 1987.

LeVinAS, Emmanuel, "Reflexiones sobre la 'técnica' fenomenológica", en Husserl. Cuadernos de Royaumont, trad. A. Podetti, Buenos Aires: Paidós, 1968.

Rodríguez, Francisco, "Carta de un positivista un fenomenólogo", Cuadernos de Filosofía Latinoamericana, vol. 27, 94 (2006) 292-308.

Spiegelberg, Herbert, The Phenomenological Movement: A Historical Introduction, The Hague: Martinus Nijhoff, 1982.

STEIN, Edith, Estrellas amarillas, Madrid: Espiritualidad, 1992.

VARGAS, Germán, Fenomenología, Formación y mundo de la vida. Problemas teóricos y metodológicos de la fenomenología, Saarbrücken: Editorial Académica Española, 2012.

-, "Respuesta a la epístola de un positivista a un fenomenólogo", Anuario Colombiano de Fenomenología, vol. I (2007) 179-194. 